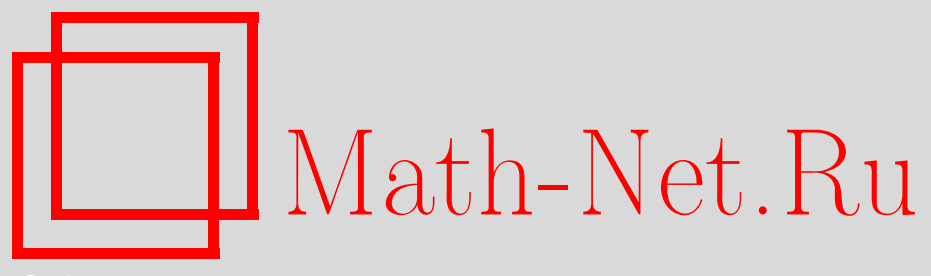

Ф. Б. Гусейнов, Б. А. Искендеров, О смешанной задаче для уравнения Баренблатта-Келтова-Кочиной в цилиндрической по пространственным переменным области, УМH, 2006, том 61, выпуск 2, 165-166

DOI: https://doi.org/10.4213/rm1706

Использование Общероссийского математического портала Math-Net.Ru подразумевает, что вы прочитали и согласны с пользовательским соглашением http://www . mathnet.ru/rus/agreement

Параметры загрузки:

IP : 54.197 .130 .99

26 апреля 2023 г., $07: 27: 05$

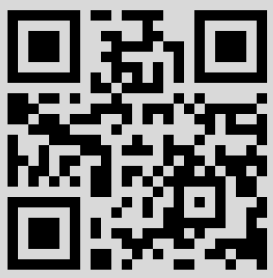




\title{
О смешанной задаче для уравнения Баренблатта-Желтова-Кочиной в цилиндрической по пространственным переменным области
}

\author{
Ф. Б. Гусейнов, Б. А. Искендеров
}

В данной работе изучена смешанная задача для уравнения Баренблатта-ЖелтоваКочиной [1] в многомерной цилиндрической области.

Пусть $R_{m}(y) \quad(m \geqslant 1)-m$-мерное евклидово пространство с элементами $y=$ $\left(y_{1}, \ldots, y_{m}\right)$, а $R_{n}(x) \quad(n \geqslant 1)$ - такое же пространство с элементами $x=\left(x_{1}, \ldots, x_{n}\right)$. Обозначим через $Ц=R_{n}(x) \times \Omega$ цилиндрическую область в $R_{n}(x) \times R_{m}(y)$, где $\Omega$ ограниченная область в $R_{m}(y)$ с достаточно гладкой границей.

Пусть $C^{(0,0,0)}(Ц \times[0, \infty))$ - пространство непрерывных в $Ц \times[0, \infty)$ функций, a $C^{(\alpha, \beta, \mu)}(Ц \times[0, \infty))$ - пространство функций, для которых $D_{x}^{\alpha} D_{y}^{\beta} D_{t}^{\mu} u(x, y, t) \in$ $C^{(0,0,0)}(Ц \times[0, \infty))$. Аналогичным образом определяется пространство $C^{(\alpha, \beta)}(Ц)$.

Рассмотрим в $Ц \times(0, \infty)$ следующую задачу:

$$
u_{t}(x, y, t)-\Delta_{n+m} u_{t}(x, y, t)=\Delta_{n+m} u(x, y, t)
$$

с начальным условием

$$
u(x, y, 0)=\varphi(x, y)
$$

и с краевым условием

$$
\left.u(x, y, t)\right|_{\partial ц}=0, \quad t>0,
$$

где $\Delta_{n+m}$ - оператор Лапласа по $(x, y), \varphi(x, y) \in C^{(2,2)}(Ц), \partial Ц=\partial \Omega \times R_{n} \times(0, \infty)$.

Обозначим через $C_{\gamma_{0}, \gamma_{1}}^{(2,2)}(Ц \times[0, \infty))$ пространство функций из $C^{(2,2,1)}(Ц \times[0, \infty))$, для которых выполняется неравенство

$$
\left\|D_{x_{j}}^{\alpha_{j}} D_{y_{\nu}}^{\beta_{\nu}} D_{t}^{\mu} u(x, y, t)\right\|_{C(L)} \leqslant C e^{-\gamma_{0} t}
$$

равномерно по $y \in \bar{\Omega}$, где $|x|$ - евклидова норма в $R_{n}, \gamma_{0}, \gamma_{1}>0$ - некоторые постоянные, $0 \leqslant \alpha_{j} \leqslant 2,0 \leqslant \beta_{\nu} \leqslant 2,0 \leqslant \mu \leqslant 1$ - целые числа и если $\alpha_{j}+\beta_{\nu} \neq 0, j=1, \ldots, n$, $\nu=1, \ldots, m$, то только одно из этих чисел отлично от нуля.

Через $H_{D}^{\nu}(\Omega) \quad(\nu \geqslant 1)$ будем обозначать подпространство пространства Соболева $H^{\nu}(\Omega)$ [2; с. 252], для элементов которого выполняются условия

$$
\left.F(x)\right|_{\partial \Omega}=0,\left.\quad \Delta F(x)\right|_{\partial \Omega}=0, \ldots,\left.\Delta^{\left[\frac{\nu-1}{2}\right]} F(x)\right|_{\partial \Omega}=0,
$$

где $[\nu]$ обозначает целую часть $\nu$.

ОПредЕлЕниЕ. Функцию $u(x, y, t)$ будем называть классическим решением задачи $(1)-(3)$, если $u(x, y, t) \in C_{\gamma_{0}, \gamma_{1}}^{(2,2,1)}(Ц \times[0, \infty)) \cap C_{\gamma_{0}, \gamma_{1}}^{(1,1,1)}(\bar{L} \times[0, \infty))$ и удовлетворяет уравнению, начальному и граничному условиям в обычном смысле.

Теорема 1. Классическое решение задачи (1)-(3) единственно, если оно сущеcmsyem.

Учитывая оценку (4), совершим преобразование Лапласа по $t$ в задаче (1)-(3). Тогда получим

$$
\begin{gathered}
k\left(1-\Delta_{n+m}\right) V(x, y, k)=\Delta_{n+m} V(x, y, k)+\varphi(x, y)+\Delta \varphi(x, y), \\
\left.V(x, y, k)\right|_{\partial Ц}=0,
\end{gathered}
$$

где $\operatorname{Re} k>0$. 
ТЕОРЕма 2. Функция Грина задачи (5)-(6) является аналитической функиией от комплексного параметра $k$ в полуплоскости $\operatorname{Re} k \geqslant 0, u$ для нее имеет место разложение

$$
\begin{aligned}
G(x, y, z, k)=\frac{i}{4} & \frac{(2 \pi)^{-\frac{n}{2}}}{k+1}|x|^{1-\frac{n}{2}} \sum_{\ell=1}^{\infty}\left(\sqrt{\lambda_{\ell}-\frac{k}{k+1}}\right)^{\frac{n}{2}-1} \\
& \times H_{\frac{n}{2}-1}^{(1)}\left(|x| \sqrt{\lambda_{\ell}-\frac{k}{k+1}}\right) \psi_{\ell}(y) \psi_{\ell}(z),
\end{aligned}
$$

где $H_{\frac{n}{2}-1}^{(1)}(z)$ - функиия Ханкеля первого рода порядка $\frac{n}{2}-1, \lambda_{\ell}-$ собственнъе значения задачи Дирихле для оператора Лапласа, $\psi_{\ell}(y)$ - соответствующие собственнье бнкиии.

При $\operatorname{Re} k \geqslant 0,|x| \geqslant \delta>0$ ряд в (7) сходится равномерно относительно $k u(x, y, z)$ в каждом компакте из Ц. Функиия Грина допускает аналитическое продолжение в полуплоскость $\operatorname{Re} k<0$ с особой точкой $k=-1$, с точками ветвления на отрицательной полуоси

$$
k_{\ell}=-\frac{\lambda_{\ell}}{\lambda_{\ell}-1}, \quad \ell=1,2, \ldots,
$$

с пределъной точкой $k=-1$.

Tеорема 3. Ecлu $\operatorname{Re} k \geqslant 0, \partial \Omega \in C^{\left[\frac{n}{2}\right]+2\left[\frac{m}{2}\right]+3}, \varphi(\xi, z) \in H_{D}^{\frac{n}{2}+2\left[\frac{m}{2}\right]+2}(\Omega) \quad \forall \xi u$

$$
\int_{R_{n}} e^{2 \gamma_{1}|\xi|}\left\|D_{\xi}^{\alpha} \varphi(\xi, z)\right\|_{H^{\frac{n}{2}+2}\left[\frac{m}{2}\right]+2}^{2} d \xi<\infty
$$

то для решения задачи (5)-(6) имеет место оценка

$$
\left\|D_{x}^{\alpha} D_{y}^{\beta} V(x, y, k)\right\| \leqslant \frac{C e^{-\gamma_{1}|x|}}{|k+1|}\left\{\int_{R_{n}} e^{2 \gamma_{1}|\xi|}\left\|D_{\xi}^{\alpha} \varphi(\xi, z)\right\|_{H^{\frac{n}{2}+2\left[\frac{m}{2}\right]+|\beta|}(\Omega)}^{2} d \xi\right\}^{\frac{1}{2}}
$$

где $C$ - константа, $0 \leqslant|\alpha| \leqslant 2,0 \leqslant|\beta| \leqslant 1, \gamma_{1}<\left|\lambda_{1}\right|^{1 / 2},|\alpha|=\alpha_{1}+\cdots+\alpha_{n}$, $|\beta|=\beta_{1}+\cdots+\beta_{m}$.

ТеОрема 4. Пусть выполняются условия теоремъ 3. Тогда для решения задачи (1)-(3) имеет место оценка

$$
\left|D_{x}^{\alpha} D_{y}^{\nu} D_{t}^{\beta} u(x, y, t)\right| \leqslant C(n) e^{-\gamma_{0} t-\gamma_{1}|t|}\left(\int_{R_{n}} e^{2 \gamma_{1}\left|\xi_{1}\right|}\left\|D_{\xi}^{\alpha} \varphi(\xi, z)\right\|_{H^{\frac{n}{2}+2}\left[\frac{m}{2}\right]+|\beta|}^{2} d \xi\right)^{\frac{1}{2}}
$$

$\left(0 \leqslant|\alpha|,|\beta| \leqslant 2, \mu=0,1 ; \gamma_{0}=\frac{\left|\lambda_{1}\right|}{\left|\lambda_{1}-1\right|}-\delta, \gamma_{1}<\left|\lambda_{1}\right|^{1 / 2}, \delta\right.$-достаточно малое число $)$.

\section{Список литературы}

[1] Г. И. Баренблатт, Ю. П. Желтов, И. Н. Кочина, Прикл. матем. мех., 24:5 (1960), 852864. [2] В. П. Михайлов, Дифференииалъные уравнения в частных производнъх, Наука, М., 1976.

Ф. Б. Гусейнов (F. B. Guseinov)

Институт математики и механики НАН Азербайджана

Б. А. Искендеров (В. А. Iskanderov)

Институт математики и механики НАН Азербайджана

E-mail: bala_iskenderov@mail.az
Представлено А. Г. Костюченко Принято редколлегией 01.12 .2005 